\title{
LIPECTOMÍA CERVICALEN TRES TIEMPOS EN UN CASO DE LIPOMATOSIS SIMÉTRICA MÚLTIPLE
}

\section{Cervical lipectomy in three times in a case of multiple symmetric lipomatosis}

\author{
Luis Miguel TORRES-MORIENTES'; Ana FERNÁNDEZ-RODRÍGUEZ; \\ María ÁLVAREZ-QUIÑONES-SANZ²; Darío MORAIS-PÉREZ ${ }^{1}$ \\ Hospital Clínico Universitario de Valladolid. ${ }^{1}$ Servicio de ORL y Patología Cérvico-Facial. ${ }^{2}$ Servicio de Anatomía \\ Patológica. Valladolid. España. \\ Correspondencia: luismitorres27@yahoo.es
}

Fecha de recepción: 28 de enero de 2018

Fecha de aceptación: 7 de marzo de 2018

Fecha de publicación: 9 de marzo de 2018

Fecha de publicación del fascículo: 1 de junio de 2019

Conflicto de intereses: Los autores declaran no tener conflictos de intereses

Imágenes: Los autores declaran haber obtenido las imágenes con el permiso de los pacientes

Política de derechos y autoarchivo: se permite el autoarchivo de la versión post-print (SHERPA/RoMEO)

Licencia CC BY-NC-ND. Licencia CreativeCommons Atribución-NoComercial-SinDerivar 4.0 Internacional

Universidad de Salamanca. Sucomercialización está sujeta al permiso del editor

RESUMEN: Introducción y objetivo: La enfermedad de Madelung en una patología rara del tejido adiposo caracterizada por el acúmulo del tejido graso no encapsulado en el cuello, hombro, tronco y extremidad proximal de miembros provocando deformidades estéticas muy llamativas en algunos casos. Método: Presentamos un caso de enfermedad de Madelung tratado en nuestro servicio que provocaba una gran deformidad estética e impotencia funcional en el paciente. Resultados: Se realizó una lipectomía cervical en tres tiempos debido a la severidad del caso para disminuir las posibles complicaciones. Discusión: La etiología de lalipomatosis simétrica múltiple es desconocida aunque existen varias hipótesis y hay asociaciones casi constantes con algunos procesos crónicos sobre todo el alcoholismo. Se han ensayado diversos tratamientos aunque el único que ha sido efectivo es la cirugía. Conclusiones: Es una entidad de etiología desconocida, con una amplia gama en el diagnóstico diferencial. La cirugía se reserva por razón estética o compromiso funcional debido a la dificultad de resección y riesgo de recidiva.

PALABRAS CLAVE: Enfermedad de Madelung; lipectomía. 


\section{LIPECTOMÍA CERVICAL EN TRES TIEMPOS EN UN CASO \\ DE LIPOMATOSIS SIMÉTRICA MÚLTIPLE \\ TORRES-MORIENTES LM ET AL}

SUMMARY: Introduction and objective: Madelung disease is a rare pathology of adipose tissue which is characterized by the accumulation of non-encapsulated fatty tissue in neck, shoulder, thorax and proximal extremity. In some cases it originates very striking aesthetic deformities. Method: We present a case of Madelung disease treated in our department that caused a great aesthetic deformity and functional impotence in the patient. Results: A cervical lipectomy was performed in three times due to the severity of the case to reduce possible complications. Discussion: The etiology of multiple symmetric lipomatosis is unknown although there are several hypotheses and almost constant associations with some chronic processes, especially alcoholism. Various treatments have been tried although the only one that has been effective is surgery. Conclusions: The etiology is unknown and the differential diagnosis includes several entities.Surgery is reserved for aesthetic reasons or functional compromise due to the difficulty of resection and risk of recurrence.

KEYWORDS: Madelung disease; lipectomy.

\section{INTRODUCCIÓN}

La enfermedad de Madelung (EM), síndrome de Launois-Bensaude o lipomatosis simétrica múltiple (LSM) es una enfermedad de etiología desconocida caracterizada por múltiples depósitos de tejido adiposo no encapsulado. Se distribuye de forma simétrica en cuello, nuca, hombros, tronco y región proximal de extremidades y respeta cara, antebrazos, piernas, manos y pies [1,2]. Fue descrita por primera vez por Brodie en 1846, seguidamente por Madelung en 1888 y 10 años más tarde LaunoisBensaude publicó 65 casos. Enzi, en 1977, acuñó el término de lipomatosis simétrica benigna (LSB), desde entonces han sido descritos en la literatura unos 200 casos [3]. Desde el punto de vista epidemiológico esta entidad predomina en varones de raza blanca con un rango de edad entre 30 y 60 años y una ratio varón /mujer entre 15-30/1. La mayor incidencia ha sido descrita en la zona del mar Mediterraneo (en Italia 1 de cada 25000 varones [3,4]. La patogénesis de la EM es desconocida aunque se sugiere una alteración del DNA mitocondrial [5]. Se ha descrito su asociación con el alcoholismo crónico en el 90\% de los casos, con alteraciones metabólicas (hiperuricemia, dislipemia, intolerancia a la glucosa), anemia macrocítica, acidosis tubular renal y polineuropatía [3]. La clínica se caracteriza por el depósito de masas múltiples simétricas de tejido adiposo no encapsulado, de crecimiento progresivo, distribuido en la región cervical, nuca, parte superior del tórax y extremidad proximal de los miembros [3,6]. En un tercio de los pacientes se asocia con ginecomastia [6].

\section{DESCRIPCIÓN}

Paciente de 58 años de edad intervenido de lipectomía cervical por enfermedad de Madelung con progresión rápida en los últimos meses con secuelas funcionales y estéticas severas. Presentaba antecedentes de hepatopatía alcohólica, fumador, hipertensión arterial (HTA) y poliposis colorectal.

En la exploración se observó una gran formación lipomatosa con deformidad estética llamativa localizada a nivel cervical anterior, lateral y posterior (Figura 1), además de un depósito graso en hombros y brazos. Se realizó tomografía computarizada (TC) observando un acúmulo simétrico graso cervical no encapsulado de gran tamaño (Figura 2).

Dadas las alteraciones y secuelas funcionales y estéticas se decidió intervenir al paciente en tres tiempos para evitar morbilidad y complicaciones importantes. Inicialmente se llevó a cabo una cervicotomía posterolateral izquierda y lipectomía cervical mediante una disección subcutánea con exéresis de dos tumoraciones adiposas de 25 y 15 $\mathrm{cm}$ de longitud. A los 4 meses debido a la buena 


\section{LIPECTOMÍA CERVICAL EN TRES TIEMPOS EN UN CASO \\ DE LIPOMATOSIS SIMÉTRICA MÚLTIPLE \\ TORRES-MORIENTES LM ET AL}

evolución se realizó una lipectomia posterolateral derecha, resecando sendas tumoraciones grasas de diámetros similares a la cirugía previa.
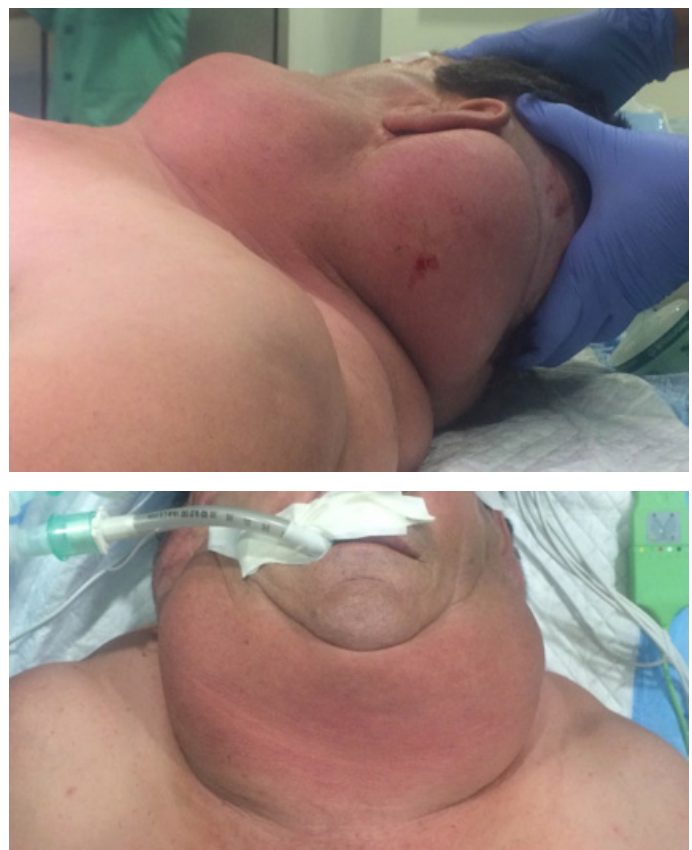

Figura 1. Deformidad cervicofacial llamativa con formaciones lipomatosas en cuello, nuca y mitad superior del tórax.

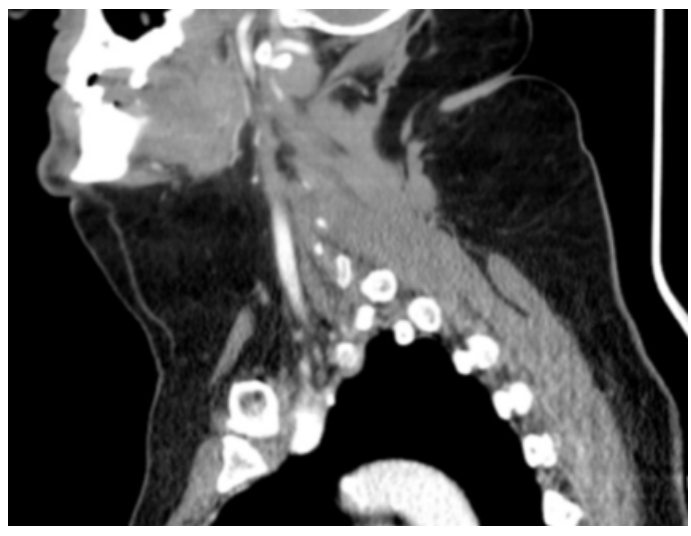

Figura 2. Corte sagital de TC donde observamos la imagen típica en «joroba de búfalo» $\mathrm{y}$ «collar de caballo».
El paciente tras ambas cirugías presentaba una necrosis leve cutánea que tras curas ambulatorias cicatriza por segunda intención (Figura 3). A los tres meses de la segunda intervención se llevó a cabo una lipectomía cervical de la nuca y región deltoidea con la colocación del paciente en decúbito prono (Figura 4).

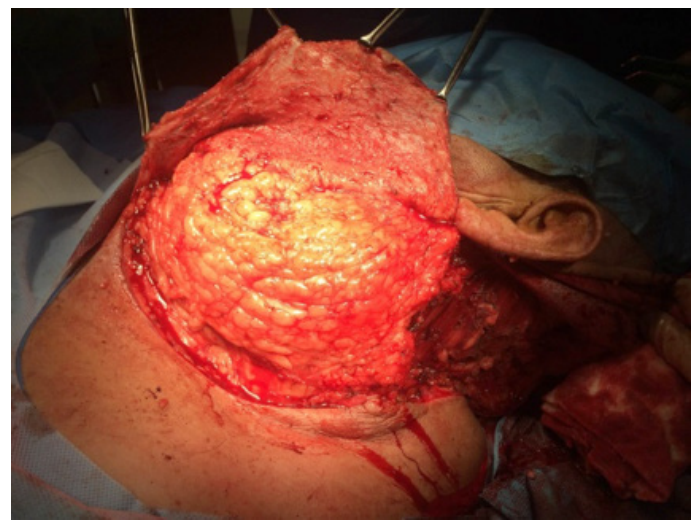

Figura 3. Cervicotomía posterolateral izquierda (primera cirugía). Acúmulo de grasa a nivel anterolateral izquierdo. Se observa la musculatura cervical posterior tras la exéresis de grasa posterior.

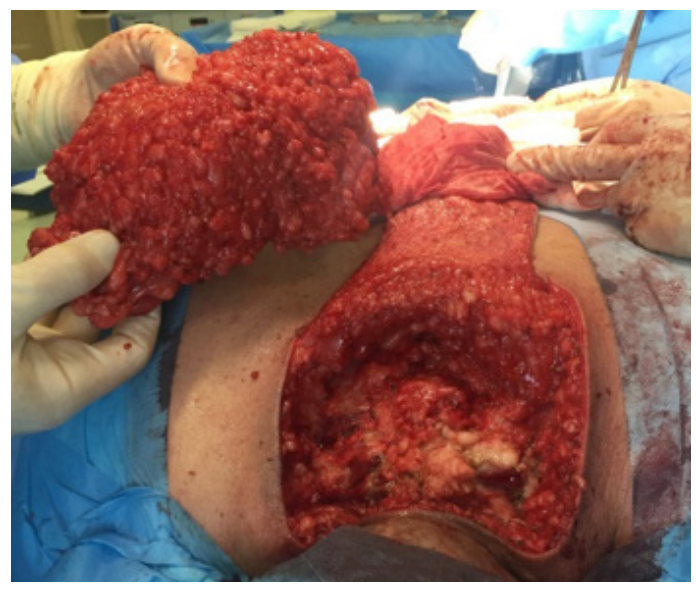

Figura 4. Colocación del paciente en decúbito prono y exéresis de acúmulo graso posterior previo colgajo con charnela inferior. 


\section{LIPECTOMÍA CERVICAL EN TRES TIEMPOS EN UN CASO \\ DE LIPOMATOSIS SIMÉTRICA MÚLTIPLE \\ TORRES-MORIENTES LM ET AL}

Tras esta cirugía presentó una necrosis cutánea de todo el colgajo posterior la cual se resolvió con curas ambulatorias y un colgajo libre llevado a cabo por el servicio de cirugía plástica (Figura 5 y 6). Salvo el compromiso vascular cutáneo de los colgajos cervicales que se resolvieron sin secuelas, el paciente no presenta ningún déficit de tipo vascular ni alteración nerviosa de pares craneales bajos. Únicamente presenta limitación funcional de las extremidades superiores y tórax debido a la progresión de la enfermedad. La anatomía patológica reveló lipomatosis (Figura 7). El paciente se encuentra estable y sin recidiva transcurrido un año de la última cirugía.

\section{DISCUSIÓN}

La EM se caracteriza por una deformidad estética significativa resultante del acúmulo de tejido adiposo simétrico de forma difusa en la región cervical, nuca, parte superior del tórax y la región proximal de las extremidades [4].

La etiología es desconocida aunque parece que está relacionado con la disfunción de un nucleótido de los adipocitos, al adenosínmonofosfato cíclico $(\mathrm{AMPc})$, y la respuesta lipolítica defectuosa a las catecolaminas, teoría defendida por Enzi et al. El alcohol también parece ser un factor importante en la hiperplasia de adipocitos a través de prolipogénesis, en individuos genéticamente susceptibles. Otros estudios sugieren la presencia de herencia mitocondrial con mutaciones puntuales del DNA mitocondrial [3,7], trastornos metabólicos que afectan al metabolismo lipídico del citocromo P450 del hígado y metabolismo de carbohidratos y consumo de esteroides [4]. Otros autores los consideran neoplasias cuyo origen sería adipocitos de grasa parda [8]. Se distinguen dos subtipos de EM; el tipo I se presenta en varones con un índice de masa corporal (IMC) normal o bajo y acumulaciones de tejido graso en cuello y hombros. Estos casos parecen estar más relacionados con antecedentes de enolismo y alteraciones metabólicas. La EM tipo
II es similar en ambos sexos con una distribución de grasa difusa e IMC más alto, similar a la obesidad simple. En ambos casos respetan antebrazos $y$ piernas $[1,7]$.
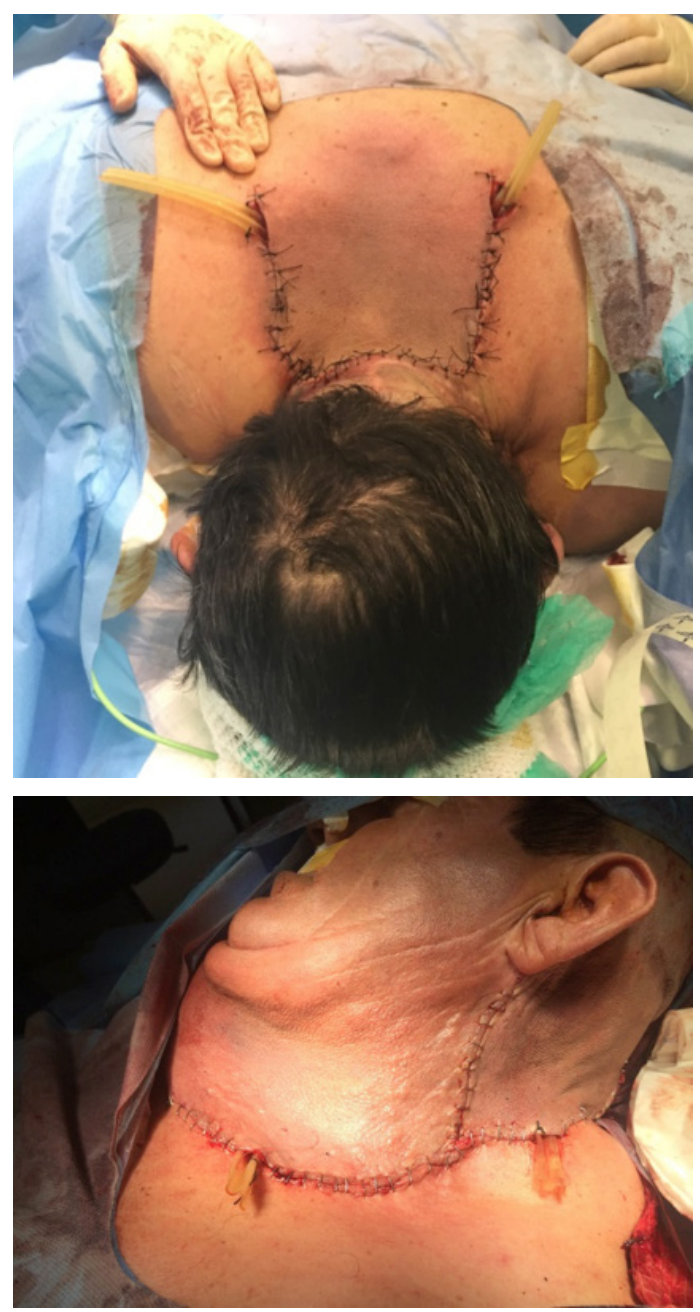

Figura 5. Imágenes de nuca y región cervical derecha tras exéresis de formaciones lipomatosas.

Desde el punto de vista clínico encontramos un depósito de grasa simétrico en distintos niveles y recuerda en la zona parotídea a las «mejillas de hámster», a nivel cervical «collar de caballo» 


\section{LIPECTOMÍA CERVICAL EN TRES TIEMPOS EN UN CASO \\ DE LIPOMATOSIS SIMÉTRICA MÚLTIPLE \\ TORRES-MORIENTES LM ET AL}

o en la nuca «joroba de búfalo». En algunas situaciones pueden limitar la movilidad de cuello y brazos e incluso llegar a producir disnea, disfagia o compresión mediastínica llegando a implicar a la vena cava o la tráquea $[1,5,7]$. En este caso únicamente presentaba una gran deformidad estética y graves secuelas funcionales derivadas de la alteración de la motilidad. Los depósitos de grasa, una vez presentes, no desaparecen espontáneamente y la enfermedad suele ser progresiva a lo largo de un periodo de años.

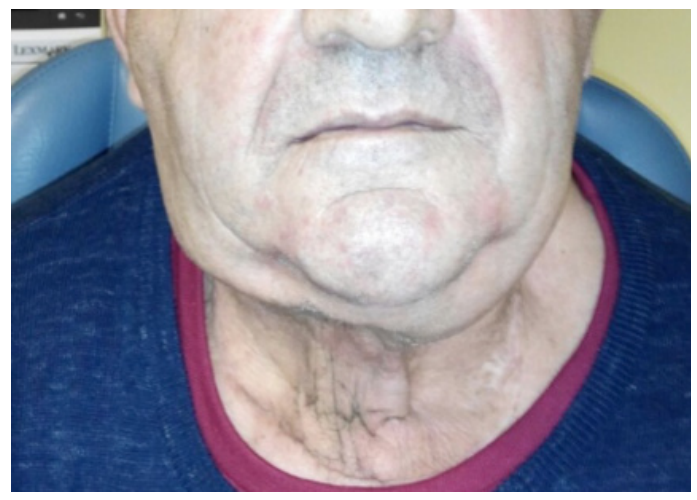

Figura 6. Imagen cervical tras meses de la última cirugía con resultado estético satisfactorio.

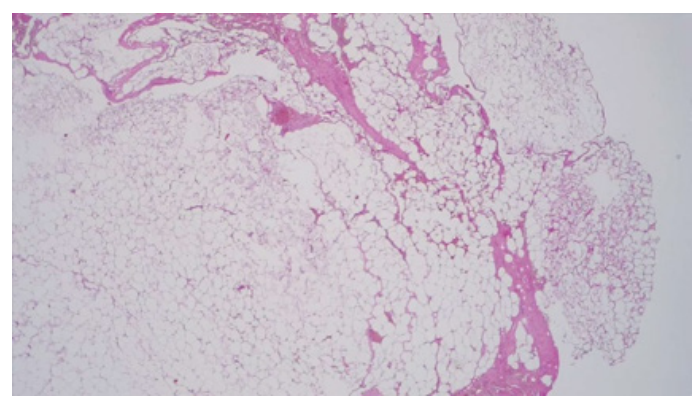

Figura 7. Tejido adiposo predominantemente maduro con septos fibrosos ( 40xHE).

Existe una asociación con anemia macrocítica y alteraciones hepáticas (60\%), síndrome metabólico (40\%), enfermedad pulmonar obstructiva crónica (23\%) e hipotiroidismo (10\%). En un $80-90 \%$ de los pacientes se observa una neuropatía periférica desmielinizante [7]. Al parecer presentan una mayor suceptibilidad para desarrollar carcinomas epidermoides en cabeza y cuello en pacientes fumadores y bebedores [9]. El riesgo de degeneración maligna a liposarcoma es excepcional.

El diagnóstico es a través de la historia clínica y el examen físico. Las pruebas complementarias como la tomografía computarizada (TC), resonancia magnética $(\mathrm{RM})$ o las pruebas de laboratorio son útiles de cara al diagnóstico diferencial, extensión de la enfermedad y para planificar un tratamiento. En la TC se observa una proliferación homogénea de tejido graso no encapsulado de bajo valor de atenuación y en la RM se observa una hiperintensidad de la señal tanto en secuencias potenciadas en T1 y T2 [2,3].

El diagnóstico diferencial incluye entidades como la lipomatosis familiar múltiple, angiolipomas, liposarcomas, enfermedad de Cushing, lipoblastomas, neurofibromatosis, distrofias musculares, síndromes linfoproliferativos [2,3].

El tratamiento en la mayoría de los casos es conservador. Se reserva el tratamiento quirúrgico en aquellos casos con limitaciones importantes o alteraciones estéticas severas [2]. La cirugía es la primera línea de tratamiento, se ha descrito la lipectomía o liposucción con muy buenos resultados aunque el riesgo principal es la recidiva por la dificultad de exéresis completa del tumor al carecer de cápsula [3,4]. En todos los casos está indicado cambios en el estilo de vida y medidas higiénicodietéticas (abstinencia alcohólica y dieta adecuada). Hay que reseñar la enorme dificultad que supone en estos pacientes llevar a cabo una traqueotomía de urgencia en situaciones de compromiso respiratorio debido al aumento de volumen adiposo cervical. Otro tratamiento descrito es la inyección de sustancias lipolíticas en el tejido celular subcutáneo con el objetivo de reducir la lipomatosis destruyendo la pared celular [4]. Todavía no se ha demostrado la efectividad de ningún tratamiento farmacológico, algunos autores recomiendan el uso 


\section{LIPECTOMÍA CERVICAL EN TRES TIEMPOS EN UN CASO \\ DE LIPOMATOSIS SIMÉTRICA MÚLTIPLE \\ TORRES-MORIENTES LM ET AL}

de B2-agonistas, no obstante la excisión quirúrgica continúa siendo el tratamiento más efectivo [10].

\section{CONCLUSIONES}

La EM se caracteriza por el acúmulo de tejido adiposo en la región cervical, mitad superior del tórax y región proximal de extremidades respetando antebrazos y piernas. La etiología es desconocida, no obstante hay varias teorías alguna relacionada con la herencia mitocondrial y en más de un $90 \%$ de los casos se relaciona con el alcoholismo. Ha llegado a relacionarse con enfermedades metabólicas y alteraciones hepáticas. La sintomatología principal es la deformidad estética simétrica llamativa llegando en algunas situaciones a impotencias funcionales que hace difícil la vida cotidiana. El tratamiento en la mayoría de los casos es observador salvo en situaciones severas donde la lipectomía o liposucción es el tratamiento electivo.

\section{BIBLIOGRAFÍA}

1. Landínez GA, Alarcos EV, Millás T, Morais D. Lipoma laríngeo asociado a enfermedad de Madelung: a propósito de un caso. Acta Otorrinolaringol Esp. 2012; 63:311-3.

2. Jiménez F, Morales JM, Corzón T. Enfermedad de Madelung. Acta Otorrinolaringol Esp.2013; 64:166-7.
3. Ikumi I, Ikuo H, Nakamoto TS, Baccan FS, Raposo LS. Multiple symmetrical lipomatosis. Braz J Otorhinolaryngol. 2014;80:90-91.

4. Andou E, Komoto M, Hasegawa T, Mizuno H, Hayashi A. Surgical excision of madelung disease using bilateral cervical lymphonode dissection technique. Its effect and the influence of previus injection lipolysis. Plast Reconstr Surg Glob Open. 2015;3:375.

5. Figueroa LA, Santiago M, González E, González A, Cáceres W, Padilla M et al. Bilateral large palpable cervical masses: not always a malignant or infectious process. Bol Asoc Med P R. 2014;106: 42-5.

6. Wollina U, Heining B. Madelung's disease. Case series and treatment by tumescent liposuction or lipectomy. Open Access Maced J Med Sci. 2017;5: 427-31.

7. Garín M, De Grado M, Argueta L. Enfermedad de Madelung. Semergen. 2012;38:211-3.

8. Segura R, Morales JM, Blanco A, Blanco A. Enfermedad de Madelung. A propósito de 6 casos. Rev Tamé 2014;3:218-22.

9. Parmar SC, Blackburn C. Madelung's disease: an uncommon disorder of unknown aetiology?. Br J Oral Maxillofac Surg. 1996;34:467-70.

10. Gabriel YA, Chew DKW, Wedderburn RV. Multiple symmetrical lipomatosis (Madelung's disease). Surgery. 2001;117-8. 